\title{
Modélisation tridimensionnelle du procédé de mise en forme électromagnétique
}

\author{
Vincent Robin $^{1}$, Éric Feulvarch ${ }^{1,2}$ et Jean-Michel Bergheau ${ }^{2, a}$ \\ 1 ESI France, ESI Group, Le Récamier, 70 rue Robert, 69458 Lyon Cedex 06, France \\ 2 Laboratoire de Tribologie et Dynamique des Systèmes, UMR 5513 CNRS/ECL/ENISE, \\ École Nationale d'Ingénieurs de Saint-Étienne, 58 rue Jean Parot, 42023 Saint-Étienne Cedex 02, France
}

Reçu le 25 mai 2007, accepté le 24 janvier 2008

\begin{abstract}
Résumé - Le procédé de formage électromagnétique, electromagnetic forming process ou EMF en anglais, consiste à déformer les métaux en appliquant une pression générée par un champ magnétique variable d'une grande intensité. L'utilisation de ce procédé en complément de la mise en forme par emboutissage d'une ébauche, permet d'accroître la quantité d'aluminium employé dans la construction automobile en améliorant la formabilité des tôles et en réduisant les coûts de production. L'objectif des travaux de recherche liés à ce procédé est d'aboutir à une meilleure compréhension du mécanisme de déformation afin de développer des équipements et des méthodes efficaces pour sa mise en œuvre industrielle. Une manière de parvenir à cet objectif est l'utilisation de la simulation numérique au travers d'une modélisation du procédé basée sur la méthode des éléments-finis. Ces travaux de développement ont été partiellement menés dans le cadre d'un projet européen appelé EMF (G3RD-CT-2002-00798).
\end{abstract}

Mots clés : Modélisation numérique / formage électromagnétique / éléments-finis

Abstract - 3D finite element modelling of electromagnetic forming process. In the electromagnetic forming process (EMF also known as magnetic pulse forming), the metal is deformed by applying a pressure generated by an intense, transient magnetic field. The EMF process in combination with deep drawing or hydroforming increases the use of aluminium for automotive components due to improved formability and cost efficient production. A great deal of research and investigation efforts are needed for gaining better understanding on the deformation mechanism in order to develop suitable forming strategy and equipment. One way to reach this target is to employ suitable FE software to model the process. This investigation was partly conducted in the framework of a European project called EMF (G3RD-CT-2002-00798).

Key words: Numerical modelling / EMF / finite-elements

\section{Introduction}

Le procédé de formage électromagnétique, electromagnetic forming process ou EMF en anglais, est un procédé de déformation des métaux par application d'une force générée par un champ magnétique variable d'une grande intensité. Un équipement électrique particulier est nécessaire pour stocker une grande quantité d'énergie électrique au travers de condensateurs, cette dernière étant libérée en une fraction de seconde pour alimenter des inducteurs par un courant de très forte intensité [1]. Le principe du procédé réside dans la création d'un

\footnotetext{
a Auteur pour correspondance : bergheau@enise.fr
}

courant induit de sens opposé dans la pièce qui génère des forces de répulsion entre l'inducteur et l'induit d'un niveau suffisant pour provoquer la déformation de ce dernier. Pour mieux contrôler la forme finale désirée, il est possible d'employer une matrice.

L'objectif des travaux de recherche liés à ce procédé est d'aboutir à une meilleure compréhension du mécanisme de déformation afin de développer des équipements et des méthodes efficaces pour sa mise en œuvre industrielle. Une manière de parvenir à cet objectif est l'utilisation de la simulation numérique au travers d'une modélisation du procédé basée sur la méthode des éléments-finis. 


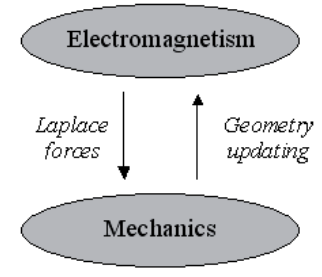

Fig. 1. Interactions entre les phénomènes électromagnétique et mécanique au cours de la mise en forme.

La première partie de cet article présente les phénomènes physiques majeurs gouvernant le procédé ainsi que leurs interactions (Fig. 1). Les niveaux de couplage requis pour simuler le procédé et les modèles numériques à mettre en œuvre sont également détaillés dans cette première partie. Dans la seconde partie, le logiciel ainsi développé est utilisé pour valider l'approche proposée sur des cas simples : tubes, plaques en aluminium. La qualité des résultats du modèle numérique met en évidence l'intérêt de la simulation pour la mise au point virtuelle du procédé et la possible extension de l'utilisation de cet outil à la modélisation numérique d'autres procédés par induction.

\section{Modélisation du couplage électromagnétique et mécanique par éléments-finis}

\subsection{Les phénomènes électromagnétiques}

Pour modéliser le procédé EMF, les phénomènes électromagnétiques gouvernés par les équations de Maxwell doivent être pris en compte. Sachant que les courants sinusoïdaux ont généralement une fréquence inférieure à $10^{12} \mathrm{~Hz}$, les courants de déplacements peuvent être négligés. Dans ce cas, en optant pour une formulation de type $A-V$ en potentiel magnétique vecteur et potentiel électrique, l'équilibre électromagnétique est régi par les équations aux dérivées partielles suivantes [2] :

$$
\begin{aligned}
\sigma\left(\frac{\partial A}{\partial t}+\operatorname{grad} V\right)+\operatorname{rot}(\nu \operatorname{rot} A) & =0 \\
\operatorname{div}\left(\sigma\left(\frac{\partial A}{\partial t}+\operatorname{grad} V\right)\right) & =0 \\
\operatorname{div} A & =0
\end{aligned}
$$

où $\sigma$ et $\nu=\frac{1}{\mu}$ représentent la conductivité électrique et la réluctance magnétique.

Les différentes conditions aux limites possibles pour le domaine d'étude $\Omega$ de frontière $\partial \Omega=\partial \Omega_{A} \cup \partial \Omega_{H}=$ $\partial \Omega_{V} \cup \partial \Omega_{j}$ sont :

- le potentiel magnétique vecteur qui peut dépendre du temps :

$$
A(t)=A_{\mathrm{d}}(t) \quad \text { sur } \quad \partial \Omega_{A}
$$

- le potentiel électrique imposé :

$$
V(t)=V_{\mathrm{d}}(t) \quad \text { sur } \quad \partial \Omega_{V}
$$

- le champ magnétique tangentiel imposé (avec $n$, normale extérieure unitaire à la surface) :

$$
H(t) \times n=H_{\mathrm{d}}(t) \quad \text { sur } \quad \partial \Omega_{H}
$$

- la densité de courant surfacique imposée :

$$
-J \cdot n=\sigma\left(\frac{\partial A}{\partial t}+\operatorname{grad} V\right) \cdot n=j_{\mathrm{d}} \quad \operatorname{sur} \quad \partial \Omega_{j}
$$

Dans l'air ainsi que dans les divers éléments constituant le procédé EMF, le traitement du problème électromagnétique par éléments-finis est basé sur la formulation variationnelle faible gouvernant $A$ et $V$, obtenue à partir des équations (1) à (7) :

$$
\forall A^{*}, A^{*}=0 \quad \text { sur } \quad \partial \Omega_{\mathrm{A}} \text { et } \forall V^{*}, V^{*}=0 \quad \text { sur } \quad \partial \Omega_{V}
$$

$$
\begin{gathered}
\int_{\Omega} A^{*} \cdot \sigma \cdot\left(\frac{\partial A}{\partial t}+\operatorname{grad} V\right) \mathrm{d} v+\int_{\Omega} \operatorname{rot} A^{*} \cdot \nu \cdot \operatorname{rot} A \mathrm{~d} v \\
+\alpha \int_{\Omega} \operatorname{div} A^{*} \cdot \operatorname{div} A \mathrm{~d} v-\int_{\partial \Omega_{\mathbf{H}}} A^{*} \cdot H^{\mathrm{d}} \mathrm{d} s=0 \\
\int_{\Omega} \operatorname{grad} V^{*} \cdot \sigma \cdot\left(\frac{\partial A}{\partial t}+\operatorname{grad} V\right) \mathrm{d} v-\int_{\partial \Omega_{j}} V^{*} \cdot j^{\mathrm{d}} \mathrm{d} s=0
\end{gathered}
$$

En appliquant une discrétisation éléments-finis, la méthode de Galerkin standard conduit à résoudre un système d'équations différentielles du premier ordre du type :

$$
\begin{aligned}
& \left\{\begin{array}{l}
\Psi_{A}(A, V) \\
\Psi_{V}(A, V)
\end{array}\right\}= \\
& \left\{\begin{array}{l}
R_{A}(A, V) \\
R_{V}(A, V)
\end{array}\right\}-\left[\begin{array}{ll}
C_{A A} & 0 \\
C_{V A} & 0
\end{array}\right] \cdot\left\{\begin{array}{l}
\dot{A} \\
\dot{V}
\end{array}\right\}=\left\{\begin{array}{l}
0 \\
0
\end{array}\right\}
\end{aligned}
$$

Les équations (10) sont résolues pas à pas en utilisant une intégration temporelle selon l'algorithme implicite d'Euler en écrivant que :

$$
A_{t}+\Delta_{t}=A_{t}+\Delta t \cdot \dot{A}_{t+\Delta t}
$$

À chaque pas de temps, la solution du problème nonlinéaire est obtenue à l'aide d'une résolution itérative de type Newton-Raphson [3].

Pour bien appréhender le caractère tridimensionnel de certains composants ou outils (notamment les inducteurs), il est parfois nécessaire de mener des analyses 3D. Les équations décrites ci-dessus ont pour cela été implémentées en 2D puis généralisées aux cas 3D dans le code de calcul SYSMAGNA ${ }^{\circledR}[4]$. 


\subsection{Les phénomènes mécaniques}

Les effets thermiques sont négligés au cours des simulations présentées dans ce travail (Fig. 1). En effet, les courants induits se concentrent en périphérie de la pièce et bien que leur intensité soit très élevée, la courte durée de l'impulsion magnétique ne génère pas une énergie suffisamment importante pour affecter le comportement mécanique de la structure dans son ensemble. De même, le travail plastique est distribué dans toute la partie déformée de la pièce et n'est pas pris en compte à la manière des hypothèses couramment admises pour la modélisation numérique des procédés d'emboutissage.

Le comportement mécanique de la pièce à mettre en forme prend en compte les fortes vitesses de déformation au travers du modèle de comportement viscoplastique de Johnson-Cook [5] :

$$
\sigma=\left(A+B\left(\varepsilon_{\mathrm{eq}}^{\mathrm{p}}\right)^{n}\right)\left(1+C \ln \dot{\varepsilon}_{\mathrm{eq}}^{*}\right)\left(1-T^{* m}\right)
$$

avec $\varepsilon_{\mathrm{eq}}^{*}=\frac{\stackrel{\bullet}{\dot{\mathrm{eq}}^{\mathrm{q}}}}{\dot{\boldsymbol{\varepsilon}}_{0}}$ et $T^{*}=\frac{\theta-T_{\mathrm{ref}}}{T_{\mathrm{melt}}-T_{\mathrm{ref}}}$

$\varepsilon_{\text {eq }}^{*}$ est le taux de déformation adimensionné avec $\dot{\varepsilon}_{0}$ généralement pris à $1,0 \mathrm{~s}^{-1}, T_{\text {melt }}, T_{\text {ref }}$ et $\theta$ représentent respectivement la température de fusion, une température de référence telle que la température ambiante et la température de la pièce. Les paramètres du modèle de Johnson-Cook ont été identifiés pour les matériaux étudiés à partir d'un dispositif expérimental mis en place à l'Université de Trento, Department of Material Engineering, en Italie.

Le problème mécanique est simulé à partir de PAMSTAMP ${ }^{\circledR}$ qui est un logiciel métier dédié à la simulation des procédés d'emboutissage [6]. Contrairement à SYSMAGNA ${ }^{\circledR}$, ce code de calcul traite les aspects mécaniques de façon explicite étant donné la rapidité des procédés d'emboutissage.

\subsection{Le couplage électromagnétique et mécanique}

Pour obtenir l'évolution de la déformée de la structure ainsi que la distribution des forces internes et des variables d'histoire au cours de la mise en forme électromagnétique, un couplage magnéto-mécanique entre SYSMAGNA ${ }^{\circledR}$ et PAM-STAMP ${ }^{\circledR}$ est réalisé.

Le principe de la simulation repose sur un découpage en macro-pas de temps au cours desquels une analyse électromagnétique et une analyse mécanique sont successivement réalisées. Les forces de Laplace sont calculées à partir des résultats issus de SYSMAGNA ${ }^{\circledR}$ en termes d'induction et de densités de courant à la fin d'un macropas de temps de calcul. Elles sont ensuite appliquées comme chargement pour la simulation mécanique réalisée dans PAM-STAMP ${ }^{\circledR}$ au cours du même macro-pas de temps. Ce processus est réitéré macro-pas de temps par macro-pas de temps afin de modéliser la totalité de la phase de mise en forme.

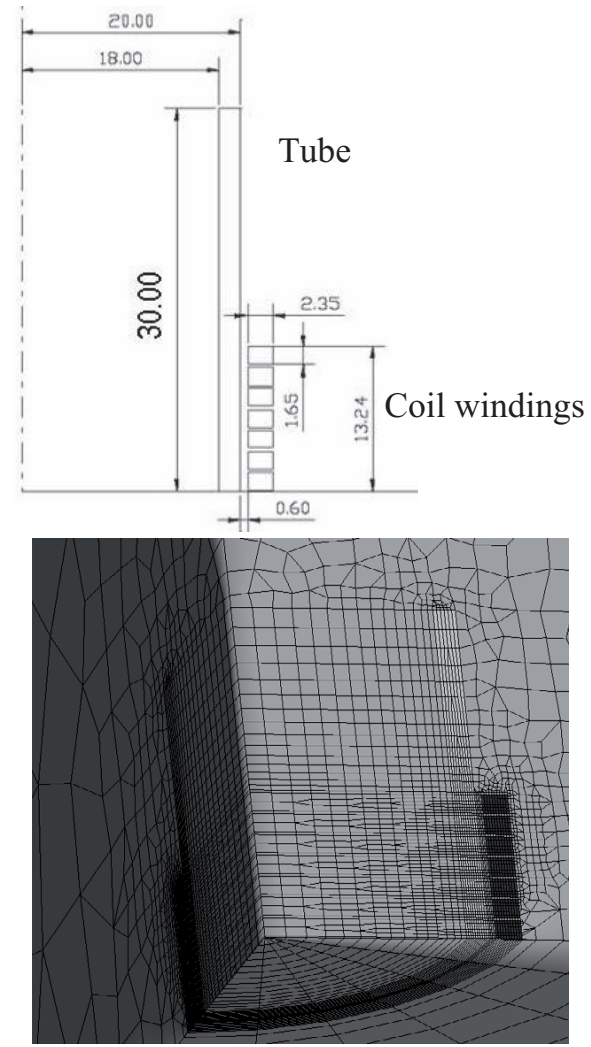

Fig. 2. Géométrie du tube en compression et maillage tridimensionnel associé.

\section{Applications}

\subsection{Essais virtuels}

Les expériences ont été conduites à l'Université de Dortmund, Department of Forming Technology, en Allemagne. Les résultats expérimentaux sont comparés à ceux obtenus par le calcul. Le comportement élastovisco-plastique avec écrouissage isotrope des matériaux utilisés est gouverné par la loi de Jonson-Cook (12). Le comportement électromagnétique des outils et de la pièce est également considéré isotrope. Aucune nonlinéarité matériau n'est prise en compte pour le calcul électromagnétique.

La mise au point d'un procédé avec ce type de configurations simples (tubes en compression ou plaques) mais représentatives d'un procédé industriel est très utile pour aider à la conception des inducteurs, à la définition des niveaux d'énergie requis ainsi qu'au choix des matériaux constituant la structure à mettre en forme.

\subsubsection{Essais sur des tubes en compression}

La géométrie (section de révolution) et le maillage de cette configuration d'essai sont présentés sur la figure 2. L'air environnant est maillé de manière à propager le champ magnétique issu des inducteurs. Le tube est constitué d'aluminium AA5754. L'énergie électrique 


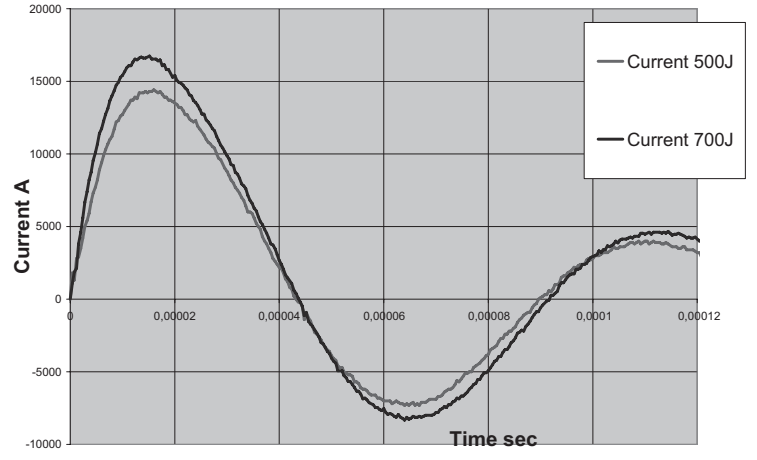

Fig. 3. Évolution du courant dans l'inducteur pour différents niveaux d'énergie de décharge électrique.

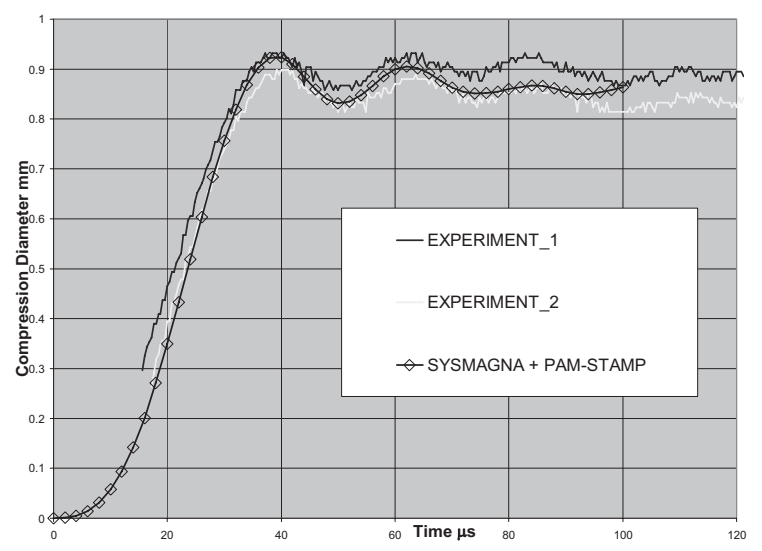

Fig. 4. Comparaison calcul/mesures de la réduction du diamètre au cours du procédé.

déchargée est de l'ordre de $500 \mathrm{~J}$. Le courant qui traverse les inducteurs devient nul après $200 \mu$ s à l'issue de la décharge (Fig. 3), c'est pourquoi des itérations de couplage entre calcul magnétique et calcul mécanique toutes les $2 \mu$ s ne sont réalisées que durant cette période.

La figure 4 fournit une comparaison entre résultats de calcul et mesures expérimentales de la réduction du diamètre au cours du temps pour plusieurs essais. Les comparaisons sont de très bonne qualité et montrent la bonne représentativité du modèle numérique et de ses données.

\subsubsection{Essais sur des plaques}

La géométrie considérée pour cette configuration d'essai est détaillée sur la figure 5. Il s'agit d'un disque d'aluminium AA5182. L'énergie déchargée par le procédé est de 1200 J. Un macro-pas de temps de couplage magnétomécanique de $2 \mu$ s est également utilisé pour conduire ces simulations. Dans cet exemple, il est nécessaire de tenir compte des contacts mécaniques qui apparaissent au cours du procédé entre la pièce et les outils de maintien et qui influencent grandement la forme conique finale de l'éprouvette. La figure 6 donne les déplacements de la surface extérieure de la plaque en fonction de la

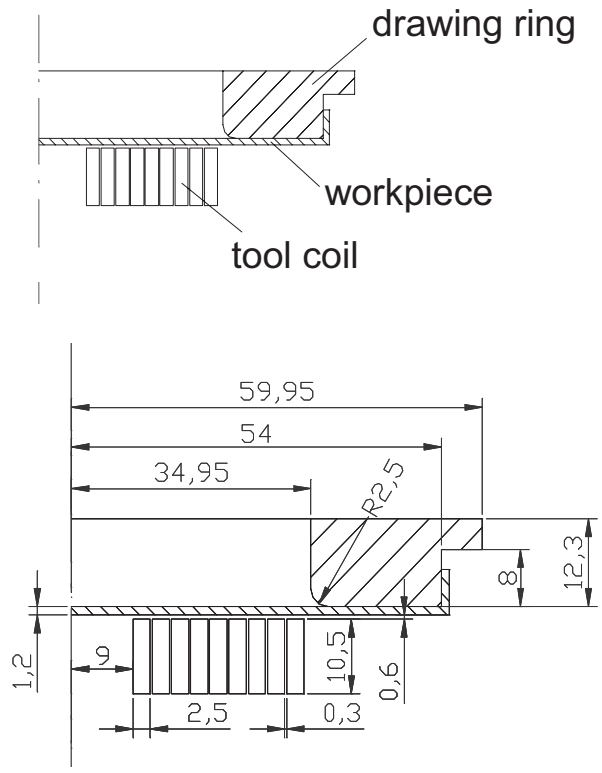

Fig. 5. Description géométrique du cas test sur des plaques.
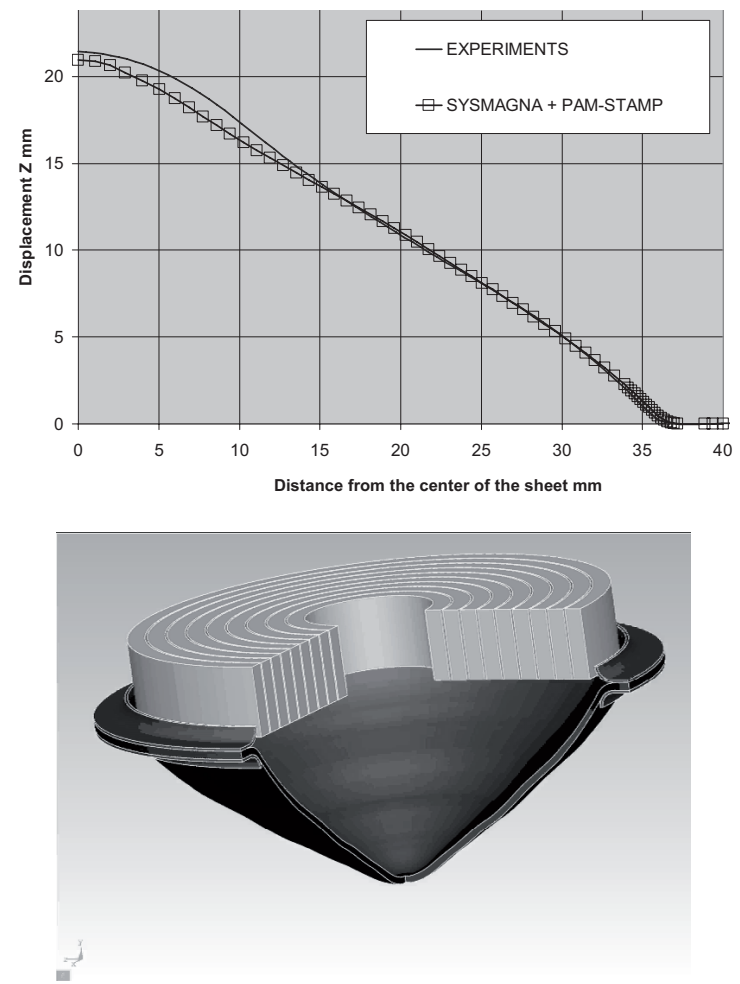

Fig. 6. Déformée du disque à l'issue du procédé.

position par rapport à son centre et la déformée finale de l'éprouvette. Pour cette configuration, modélisation numérique et expériences révèlent une nouvelle fois une excellente corrélation. 

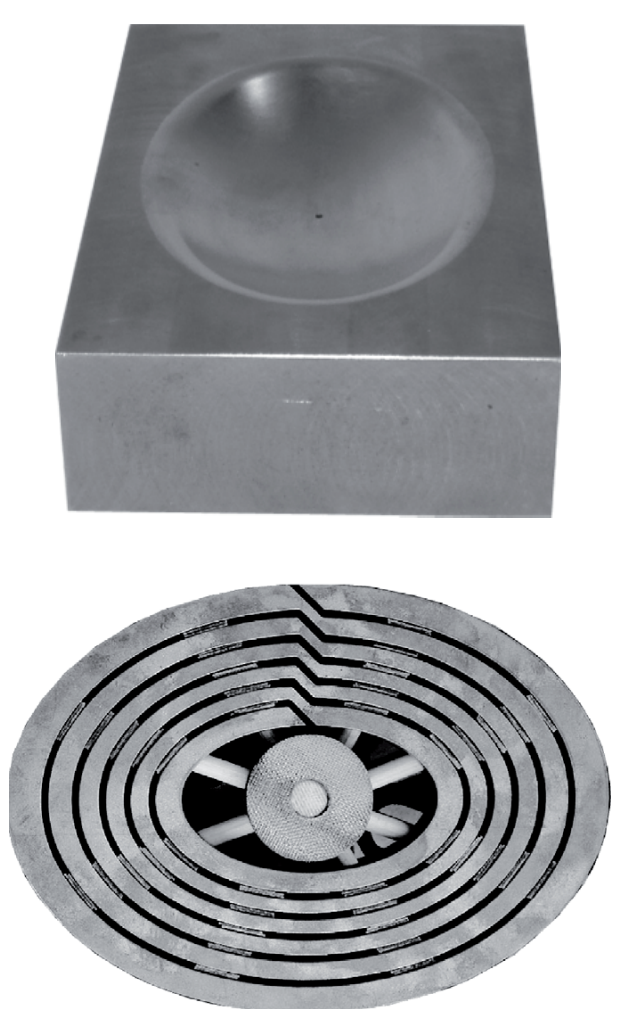

Fig. 7. Images de la matrice et de l'inducteur dans le cadre de l'application industrielle.

\subsection{Application industrielle}

Cette section permet d'illustrer l'ensemble des fonctionnalités de l'outil numérique de modélisation du procédé par formage électromagnétique car le cas proposé ne présente pas de symétrie de révolution. Les analyses électromagnétiques et mécaniques sont réalisées en 3D. Il s'agit d'une plaque en aluminium AA6016 que l'on souhaite mettre en forme dans une matrice ovale (Fig. 7). Cette forme est l'ébauche d'un renfoncement destiné aux poignées des portières de voiture. Il est en effet délicat de déformer cette zone lors de l'emboutissage de la portière complète et l'utilisation du procédé EMF, lors d'une passe de finition, offre une alternative intéressante. Deux énergies de décharge électrique ont été testées et comparées avec les résultats des calculs éléments-finis. Dans le cas de l'énergie la plus élevée, soit $2100 \mathrm{~J}$, la forme finale n'est pas parfaite comme le montre la figure 8 . La figure 9 montre que les résultats expérimentaux, qui permettent d'obtenir une bonne forme finale de la pièce avec une énergie de $1200 \mathrm{~J}$, sont retrouvés par l'analyse numérique.

\section{Conclusion et perspectives}

Un des objectifs de ce projet était de participer au développement industriel du procédé EMF par la

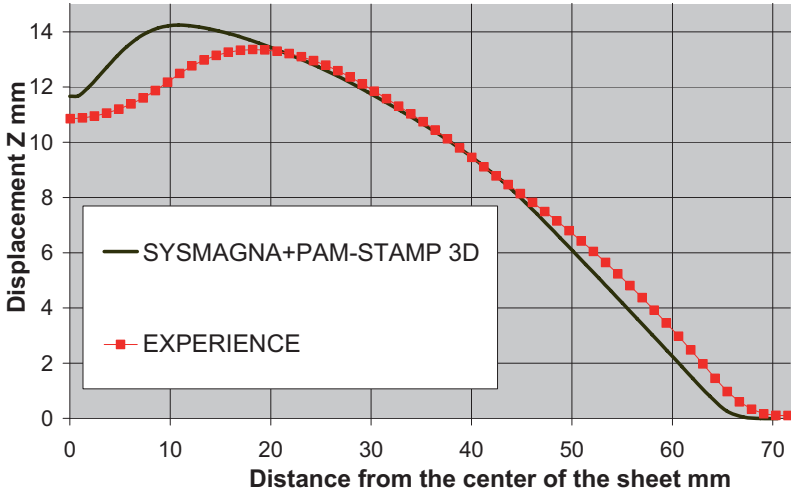

Fig. 8. Cas avec énergie de $2100 \mathrm{~J}$ : la tôle déformée n'atteint pas la forme escomptée.
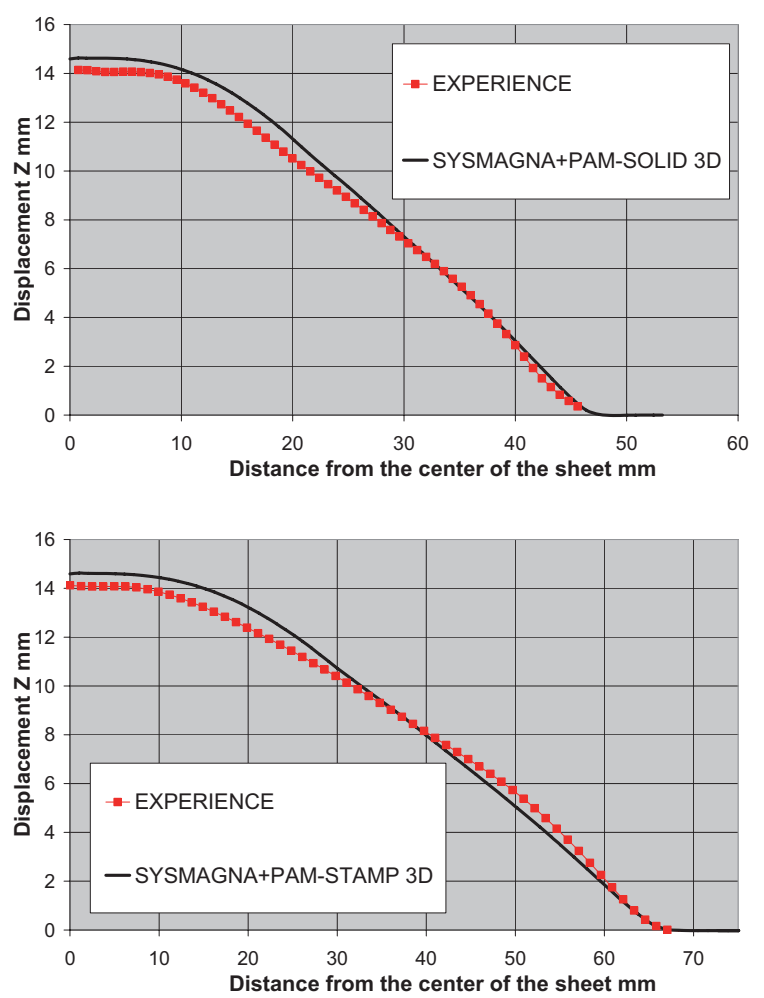

Fig. 9. Cas avec énergie de $1200 \mathrm{~J}$ : déformée finale selon le grand axe et le petit axe - comparaisons calculs/mesures.

mise au point d'outils de simulation numérique permettant de faciliter la définition du process : outillages, paramètres... La qualité des résultats des calculs par rapport aux mesures expérimentales montre la performance des modèles électromagnétique et mécanique développés, la validité des hypothèses choisies ainsi que la pertinence des données matériau. De plus, les temps de calcul des castests présentés dans cet article, qui sont bien inférieurs à l'heure sur un ordinateur personnel récent, permettent de tester en série ou parallèlement plusieurs configurations pour mettre au point virtuellement le procédé. Cependant une des difficultés de ce type d'application est le maillage 


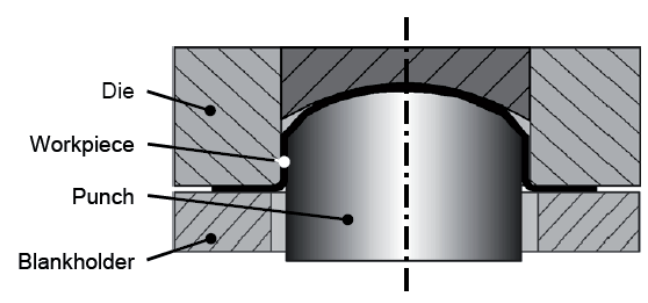

Courtesy Poynting GmbH and Institut für Umformtechnik und Leichtbau
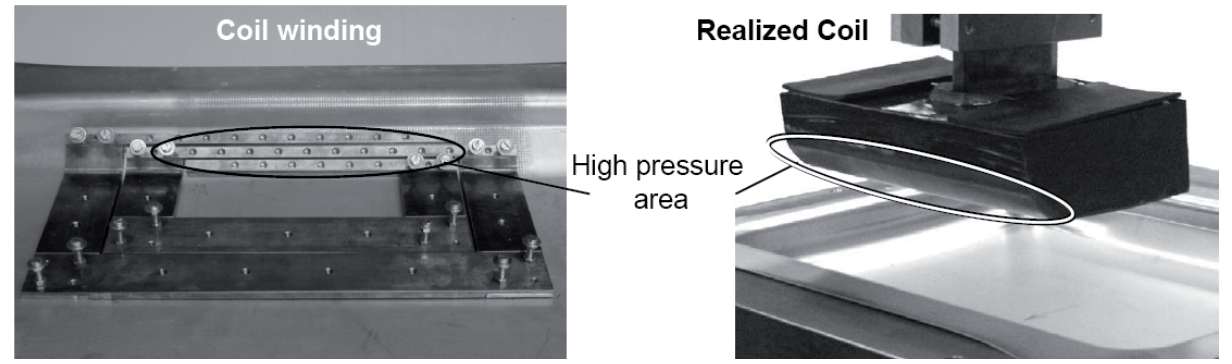

Fig. 10. Mise en forme de finition des congés par le procédé EMF après emboutissage - principe et mise en ouvre.
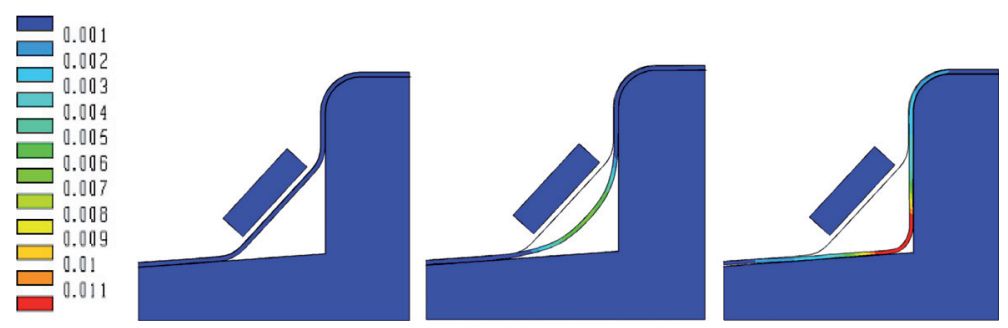

Fig. 11. Évolution de la déformée (isovaleurs de la norme du déplacement en m) selon une section au cours de la mise en forme électromagnétique de finition d'un congé.

de l'air qui dans le cas de pièces mobiles peut nécessiter un remaillage pour conserver une qualité constante de la forme des éléments. Une méthode pour prévenir ce remaillage est l'utilisation des éléments de frontières pour représenter l'environnement ambiant [7]. Ce type d'approche, dont la mise en équation n'est pas détaillée ici, a été utilisé avec succès pour la modélisation numérique de la passe de finition de la mise en forme d'un congé après emboutissage (Figs. 10 et 11). L'un des principaux inconvénients d'un couplage éléments-finis/éléments de frontière est la formation de matrices pleines liant les degrés de libertés des nœuds de la frontière du domaine éléments-finis. Cela conduit à des encombrements mémoire et des temps de résolution importants dans le cas d'analyses tridimensionnelles. D'autres méthodologies peuvent être envisagées pour parvenir à surmonter ces difficultés selon les procédés par induction à modéliser : le maillage et remaillage de l'air, la méthode des superéléments. . .

Ce travail offre des perspectives intéressantes d'application industrielle pour la simulation des procédés par induction comme le pliage électromagnétique (hemming en anglais) ou encore le soudage par impulsion magnétique.

Remerciements. Nous tenons à remercier l'ensemble des partenaires du projet européen EMF (G3RD-CT-2002-00798) pour leur collaboration constructive à la réussite du projet et également la Commission européenne pour son soutien financier à nos travaux de recherche et développement.

\section{Références}

[1] V. Psyk, C. Beerwald, M. Kleiner, M. Beerwald, A. Henselek, Use of electromagnetic forming in process combination for the production of automotive part, Proceedings of the 2nd European Pulser Power Symposium, Hamburg, Germany, 2005

[2] J.-L. Coulomb, Analyse tridimensionnelle des champs électriques et magnétiques par la méthode des éléments finis, Ph.D. Thesis, Thèse d'État, 1981

[3] Ph. Conraux, M. Pignol, V. Robin, J.M. Bergheau, 3D finite element modelling of electromagnetic forming processes, Proceedings of the 2nd International Conference on High Speed Forming, Dortmund, Germany, 2006

[4] SYSMAGNA ${ }^{\circledR}$, Manuel utilisateur, ESI Group 2007

[5] G.R. Johnson, W.H. Cook, A constitutive model and data for metals subjected to large strains, high strain rates and high temperature, Proceedings of the 7th International Symposium on Ballistic, 1983, pp. 541-547

[6] PAM-STAMP ${ }^{\circledR}$, Manuel utilisateur, ESI Group 2007

[7] R. Pascal, P. Conraux, J.M. Bergheau, Coupling between finite elements and boundary elements for the numerical simulation of induction heating processes using an harmonic balance method, IEEE Trans. Magnetics, special CEFC issue, 39 (2003) 1535-1538 